\title{
Persuasion Tactics in the Implementation of Large-Scale EHR Suites in Public Healthcare
}

\author{
Gunnar ELLINGSEN ${ }^{\mathrm{a}, 1}$, Bente CHRISTENSEN ${ }^{\mathrm{b}}$ and Morten HERTZUM ${ }^{\mathrm{c}}$ \\ a UiT - The Arctic University of Norway, Tromsø, Norway \\ ${ }^{\mathrm{b}}$ Nord University, Bodø, Norway \\ ${ }^{\mathrm{c}}$ University of Copenhagen, Copenhagen, Denmark
}

\begin{abstract}
Large-scale electronic health record (EHR) suites have the potential to cover a broad range of use needs across various healthcare domains. However, a challenge that must be solved is the distributed governance structure of public healthcare: Regional health authorities regulate hospitals, municipalities are responsible for first-line healthcare services, and general practitioners (GPs) have an independent entrepreneurial role. In such settings, EHR program owners cannot enforce municipalities and GPs to come on board. Thus, we examine what tactics owners of large-scale EHR suite programs apply to persuade municipalities to participate, how strongly these tactics are enforced, and the consequences. Empirically, we focus on the Health Platform program in Central Norway where the goal is to implement the U.S. Epic EHR suite in 2022. Theoretically, the paper is positioned in the socio-technical literature.
\end{abstract}

Keywords. EHR suites, Epic, persuasion tactics, pressure, socio-technical, technological determinism

\section{Introduction}

The overall goal for large-scale electronic health record (EHR) suites is to cover a broad range of use scenarios for healthcare workers in hospitals, nursing homes, home-care services, and general practitioner (GP) clinics. Such a wide scope implies that the EHR suite can be used as the primary system in entire healthcare regions to ensure a seamless information flow among the various domains. This is supposed to provide quality and safety in treatment and care of all steps of a patient's pathway throughout the healthcare service.

However, a challenge that must be solved is the distributed governance structure of public healthcare (as in the Scandinavian countries): Regional health authorities regulate hospitals, municipalities are responsible for first-line healthcare services, and GPs have an independent entrepreneurial role. In such settings, EHR program owners (typically anchored in regional health authorities) cannot force municipalities and GPs to come on board, but must rely on willingness and persuasion tactics. This is not straightforward given the scale and risk of such projects as well as the diversity of the stakeholders. Thus,

${ }^{1}$ Gunnar Ellingsen, Corresponding author, UIT- The Arctic University of Norway, research group: SOF NORD, 9038 Tromsø, Norway; E-mail: gunnar.ellingsen@uit.no. 
we ask the following research question: What tactics do owners of large-scale EHR suite programs apply to persuade municipalities to participate, how strongly are these tactics enforced, and what are the consequences?

Empirically, we focus on the Health Platform program in Central Norway where the goal is to implement the U.S. Epic EHR suite in 2022 [1,2]. The owners of the program (the Central Norway Regional Health Authority and Trondheim municipality) face the challenge of persuading the 64 municipalities and the many GPs in the region to participate. Although many municipalities have decided to participate, others are exploring alternatives. Theoretically, this paper is positioned in the socio-technical literature, meaning that technology always must be understood in its social context [3].

\section{Methods}

We adhere to an interpretive research approach, which considers a phenomenon from a variety of viewpoints [4,5]. In particular, we contrast the overall aim of the Health Platform program with the responses of three municipality consortia considering whether to participate in the Health Platform program.

The data collection consists of three one-hour interviews with key informants during spring 2021. The informants represent three different municipality consortia in Central Norway, each consisting of 6 to 12 municipalities. We label informants coordinators due to their long professional experience with EHRs and their role in advising directors, politicians, and municipal councils about the Health Platform program and possible alternatives. In one interview, two informants participated. In the text, we refer to the informants as Coordinator 1 to Coordinator 4. The first and second authors conducted and recorded the interviews over Zoom. We include six interviews conducted with Health Platform managers in 2018, nine interviews with GPs in Central Norway in 2019, and interviews with 10 healthcare professionals in Tromsø municipality during autumn 2020 as background sources. All interviews were transcribed for analysis.

\section{The Health Platform program and municipalities in Central Norway}

The Health Platform is a regional program owned by the Central Norway Regional Health Authority and Trondheim municipality. It is also a pilot for the national goal of “one citizen - one record". In 2019, the program signed a NOK 2.7 billion (EUR 270 million) contract with Epic Systems Corporation to acquire and implement the Epic EHR suite in the whole region, including all hospitals, GP clinics, nursing homes, and homecare services. The number of healthcare workers in the region totals around 44,000 [1,2].

A suite system, Epic is largely self-contained. Most of the functionalities needed by health personnel are supposed to be provided by Epic, either as ready-for-use functionality or through configuration. However, because for the most part Epic is a hospital-oriented system, some functionality for municipalities must be developed, for instance, related to case management, medication, and welfare technology integration.

The Central Norway Regional Health Authority and Trondheim municipality will start implementing Epic in the three regional hospitals and in Trondheim municipality in 2022. Subsequently, the implementation process will continue in the other municipalities. Health Platform management has pointed out that a crucial point in the preparations is to ensure that the municipalities buy in to the Epic solution as soon as possible to create 
stability and predictability for the program. If more municipalities and GPs participate, the region will realize more benefit from the Epic EHR suite.

Similar to municipalities in the rest of Norway, the 64 municipalities in Central Norway are the lowest administrative and politically elected level. They are responsible for providing first-line healthcare services to citizens in the local community. This responsibility includes managing nursing homes and home-care services, as well as providing public health services. Municipalities also have binding agreements with GPs. When specialized treatment is needed, citizens are referred to tertiary care facilities, i.e., one of the three hospital trusts in the region, which (as in the rest of Norway) are governed by the state through a regional health authority.

For the hospitals in the region, replacing their current EHR, which they share, is a pressing matter. For the municipalities, however, the situation is slightly different. Although their current systems in nursing homes and home-care services (Profil, Gerica and Cosdoc) lack up-to-date functionality and well-developed integration capabilities, there is no urgent need to replace them. Similarly, GPs have quite modern systems with which they are satisfied.

Despite this, the Health Platform management emphasized from early in the project that the goal of the program is not necessarily to meet each and every specific requirement of each user domain. The goal is to create a comprehensive health service that connects hospitals, nursing homes, home-care services, and GP clinics. The aim is to provide all health personnel — and patients - with complete and up-to-date information about the patients' condition and medication. Many municipalities responded very positively to this aim and indicated immediately that they would participate in the program.

However, other municipalities have increasing concerns. A key issue is that the municipalities do not know the specific functionality they will get until they go live, because much of the software for the municipalities still must be developed. In addition, integration with the national welfare technology hub, which enables integration between EHRs and welfare technologies in the municipalities, will not be delivered in the first round. Moreover, as go-live approaches, politicians in the municipalities are increasingly realizing that transitioning to Epic will require a huge effort, and the investment and operating costs will total much more than the cost of the municipalities' current EHRs. Therefore, some municipalities are exploring alternatives. One alternative is the so-called "zero-alternative," which implies continuing to use their current EHR systems. Upgrading these systems with national integration modules may facilitate the flow of information among the use domains; for example, a national shared medication list for patients is being tested in another health region. Essentially, this alternative is about establishing interoperability between the systems and to focus on standards that vendors should adhere to.

The Health Platform program and Trondheim municipality reacted immediately to the exploration of the zero-alternative. According to informants, it instigated "frenetic" meeting activity between them and the potential "breakout municipalities" to convince these municipalities that Epic is the better alternative. The owners have, according to informants, stated the benefits of the Epic EHR suite quite strongly, for example, arguing that: "It is the only alternative for a future-oriented EHR." "It will revolutionize the healthcare service." "It is a choice between the Stone Age and the 21st century."

According to our informants, to motivate municipalities to opt in, there is "enormous hype" about the benefits of sharing information. This hype has influenced some municipalities. One informant said, "My colleagues in other municipalities say that the 
municipalities have no choice; we just have to do this, and we are stupid if we do not join because then we will be left behind" (Coordinator 1).

For some of the municipalities that have opted in, the exploration of the zeroalternative is a nuisance. In response to a presentation of Epic and the zero-alternative, Coordinator 2 stated that a municipal health director said, "I don't understand why you are talking about these alternatives; it is just confusing. We shall have Epic."

Many municipal councilors and politicians are also motivated by a sense of community and belonging. They consider the Health Platform program a Central Norway (Norwegian: "Trøndersk") project and argue that they should stick with the other Central Norway municipalities. Moreover, some act out of blind solidarity with Trondheim municipality because it is the largest municipality in the region. According to one informant, other municipal directors find the program overwhelming. These directors do not think that their municipalities have the competence and resources to participate. Furthermore, these directors have problems raising these concerns in public, i.e., in the county municipal director committee. If they do, they will be blamed for not being in step with the rest of the region.

Given the size and ambition of the program, there are concerns about whether the municipalities have the necessary competences. Therefore, those that are undecided appreciate a careful assessment of the alternatives. The lack of sufficient competence in the municipalities combined with considerable pressure to opt in mean that some municipalities may wake up to an unpleasant surprise after implementation, as one informant put it.

Traditionally, the acquisition of EHRs has had a strong foothold in the professional practices with deep knowledge of use scenarios, technologies, and users, but the political dimension of the Health Platform program has become more explicit. Coordinator 4 said, "The Health Platform program has become politics. It is really very strange; we must not forget the users who are going to work with this." Coordinator 1 argued along the same lines: "When municipal directors and councilors involve themselves in the purchase of subject matter systems, all the warning lights should flash; we have never experienced that before. Then the end-user perspective is no longer in focus, and other concerns have taken precedence." It is telling then that Trondheim municipality will not have a finalized system when they go live. This is very unusual, as Coordinator 3 pointed out: "Trondheim municipality is quite brave to start first and implement Epic before it is fully developed. We dare not be so forward-leaning."

Not surprisingly, the owners are very active in interaction networks, and arenas for promoting the Health Platform program, because they have a strong interest in getting the municipalities on board. These arenas include those run by the Norwegian Association of Local and Regional Authorities and the County Governor. Coordinator 2 said, "I am puzzled that both the Norwegian Association of Local and Regional Authorities and the County Governor let their arenas be used for this interest exclusively. As a result, this makes it difficult for the municipalities to withstand the pressure, and it is difficult for them to see that there are any alternatives."

\section{Concluding discussion}

We contend that the recent tactics to persuade municipalities to participate have taken technology deterministic [6] proportions. Epic is presented as the only alternative, and the municipalities' participation is largely taken for granted. From early in the project, 
the municipalities' participation has been considered a condition for success in the Health Platform program, i.e., to create a comprehensive health service in Central Norway. Therefore, the recent reservations among some municipalities - manifested through the exploration of the zero-alternative - threatens the program's success. This puts pressure on the Health Platform program to persuade the municipalities to opt in, apparently through increasingly stronger slogans, such as "this is the future," "not falling behind," and "revolutionizing healthcare." The tactics also put pressure on the municipalities that want to explore the zero-alternative before they decide what to do. This suggests that opting in has never been a real option. The municipalities' participation has largely been taken for granted, and some municipalities are now asking themselves, what is the consequence of opting out?

Presently, there is a strong "alliance" [7] among the Health Platform, Trondheim municipality, and the municipalities that have opted in. In this alliance, participation translates into "solidarity" with Central Norway and with the municipalities that have decided to implement Epic. Through the possibility to promote the Health Platform on their arenas, the alliance has also received approval from the Norwegian Association of Local and Regional Authorities and the County Governor. Obviously, such a strong network is hard to withstand, although the presence of the zero-alternative provides some counterweight.

However, the combination of a large-scale system, promotion efforts characterized by technology determinism, and a lack of competence in the municipalities creates an unpredictable cocktail. The sheer size of Epic questions whether there is sufficient professional competence in the municipalities to handle the implementation and future operation. In addition, given how the Health Platform program has increasingly turned "political," municipal politicians have been heavily involved in decision-making processes that normally are run by professional users and subject matter specialists. The consequence is that decisions may be made without proper professional advice. It is telling that the municipalities are asked to buy in to a system that they have not yet seen in real life. This is a glaring deviation from traditional municipal purchasing practice, typically characterized by caution and frugality.

\section{References}

[1] M. Hertzum, G. Ellingsen, The implementation of an electronic health record: Comparing preparations for Epic in Norway with experiences from the UK and Denmark, International Journal of Medical Informatics 129 (2019), 312-317.

[2] G. Ellingsen, M. Hertzum, User requirements meet large-scale EHR suites: Norwegian preparations for Epic, in L. Pape-Haugaard, C. Lovis, I.C. Madsen, P. Weber, P.H. Nielsen, P. Scott (Eds.), Digital Personalized Health and Medicine. Proceedings of MIE, IOS Press, Geneva, (2020), 1-5.

[3] P.M. Leonardi, Crossing the implementation line: The mutual constitution of technology and organizing across development and use activities, Communication Theory 19 (2009), 278-310.

[4] H. Klein, M. Myers, A set of principles for conducting and evaluating interpretive field studies in information systems, MIS Quarterly 23 (1999), 67-94.

[5] G. Walsham, Interpretive case studies in IS research. Nature and method, European Journal of Information Systems 4 (1995), 74-81.

[6] S. Wyatt, Technological determinism is dead; long live technological determinism, in E.J. Hackett, O. Amsterdamska, M. Lynch, J. Wajcman (Eds.), The Handbook of Science and Technology Studies, The MIT Press, London, (2008), 165-180.

[7] B. Latour, Reassembling the Social. An Introduction to Actor-Network-Theory. Oxford University Press, Oxford, 2005. 